\title{
Casimir wormholes
}

\author{
Remo Garattini ${ }^{1,2, a_{(D}}$ \\ ${ }^{1}$ Dipartimento di Ingegneria e Scienze Applicate, Università degli Studi di Bergamo, Viale Marconi 5, 24044 Dalmine, Bergamo, Italy \\ 2 I.N.F.N., Sezione di Milano, Milan, Italy
}

Received: 9 July 2019 / Accepted: 7 November 2019 / Published online: 20 November 2019

(C) The Author(s) 2019

\begin{abstract}
Casimir energy is always indicated as a potential source to generate a traversable wormhole. It is also used to prove the existence of negative energy which can be built in the laboratory. However, in the scientific literature there is no trace of the consequences on the traversable wormhole itself. In this work, we would like to consider such a source to see if and which kind of traversable wormhole can be produced. In a further analysis, we examine also the consequences of quantum weak energy conditions on the traversability of the wormhole. We find agreement with the original Casimir traversable wormhole. Nevertheless, despite the traversability result, one finds once again that the traversability is only in principle but not in practice.
\end{abstract}

\section{Introduction}

The Casimir effect appears between two plane parallel, closely spaced, uncharged, metallic plates in vacuum. It was predicted theoretically in 1948 [1] and experimentally confirmed in the Philips laboratories [2-4]. However, only in recent years further reliable experimental investigations have confirmed such a phenomenon [5,6]. The interesting feature of this effect is that an attractive force appears which is generated by negative energy. Indeed the attractive force arises because the renormalized energy assumes the following form:

$E^{\operatorname{Ren}}(a)=-\frac{\hbar c \pi^{2} S}{720 a^{3}}$,

where $S$ is the surface of the plates and $a$ is the separation between them. The force can be obtained with the computation of

$F(a)=-\frac{d E^{\operatorname{Ren}}(a)}{d a}=-3 \frac{\hbar c \pi^{2} S}{720 a^{4}}$,

a e-mail: remo.garattini@unibg.it producing also a pressure

$P(a)=\frac{F(a)}{S}=-3 \frac{\hbar c \pi^{2}}{720 a^{4}}$.

It is immediate to recognize that the energy density is nothing but

$\rho_{C}(a)=-\frac{\hbar c \pi^{2}}{720 a^{4}}$,

suggesting the existence of a relation between the pressure $P$ and the energy density $\rho$ described by an equation of state (EoS) of the form $P=\omega \rho$ with $\omega=3$. The nature of this effect is connected with the zero point energy (ZPE) of the quantum electrodynamics vacuum distorted by the plates. It is important to observe that this effect shows a strong dependence on the geometry of the boundaries. Indeed, Boyer [7] proved the positivity of the Casimir effect for a conducting spherical shell of radius $r$. The same positivity has been proved also in Ref. [8], by means of heat kernel and zeta regularization techniques. To the best of our knowledge, the Casimir energy represents the only artificial source of exotic matter realizable in a laboratory. ${ }^{1}$ Exotic matter violates the null energy condition (NEC), namely for any null vector $k^{\mu}$, we have $T_{\mu \nu} k^{\mu} k^{\nu} \geq 0$. Violation of the NEC is related to the existence of a bizarre but amazing object predicted by General Relativity: a traversable wormhole. Traversable wormholes (TWs) are solutions of the Einstein's field equations (EFEs) powered by classical sources [10,11]. However, given the quantum nature of the Casimir effect, the EFE must be replaced with the semiclassical $\mathrm{EFE}$, namely

$G_{\mu \nu}=\kappa\left\langle T_{\mu \nu}\right\rangle^{\text {Ren }} \quad \kappa=\frac{8 \pi G}{c^{4}}$,

\footnotetext{
1 Actually, there exists also the possibility of taking into consideration a squeezed vacuum. See for example Ref. [9].
} 
where $\left\langle T_{\mu \nu}\right\rangle^{\text {Ren }}$ describes the renormalized quantum contribution of some matter fields: in this specific case, the electromagnetic field. To establish a connection between exotic matter and a TW, we introduce the following spacetime metric:

$d s^{2}=-e^{2 \phi(r)} d t^{2}+\frac{d r^{2}}{1-b(r) / r}+r^{2} d \Omega^{2}$,

representing a spherically symmetric and static wormhole. $d \Omega^{2}=d \theta^{2}+\sin ^{2} \theta d \phi^{2}$ is the line element of the unit sphere. $\phi(r)$ and $b(r)$ are arbitrary functions of the radial coordinate $r \in\left[r_{0},+\infty\right)$, denoted as the redshift function, and the shape function, respectively $[10,11]$. A fundamental property of a wormhole is that a flaring out condition of the throat, given by $\left(b-b^{\prime} r\right) / b^{2}>0$, must be satisfied as well as the requirement that $1-b(r) / r>0$. Furthermore, at the throat $b\left(r_{0}\right)=r_{0}$, and the condition $b^{\prime}\left(r_{0}\right)<1$ is imposed to have wormhole solutions. It is also fundamental that there are no horizons present, which are identified as the surfaces with $e^{2 \phi} \rightarrow 0$, so that $\phi(r)$ must be finite everywhere. With the help of the line element (6), we can write the EFE in an orthonormal reference frame, leading to the following set of equations:

$$
\begin{aligned}
& \frac{b^{\prime}(r)}{r^{2}}=\kappa \rho(r), \\
& \frac{2}{r}\left(1-\frac{b(r)}{r}\right) \phi^{\prime}(r)-\frac{b(r)}{r^{3}}=\kappa p_{r}(r), \\
& \left\{\left(1-\frac{b(r)}{r}\right)\left[\phi^{\prime \prime}(r)+\phi^{\prime}(r)\left(\phi^{\prime}(r)+\frac{1}{r}\right)\right]\right. \\
& \left.-\frac{b^{\prime}(r) r-b(r)}{2 r^{2}}\left(\phi^{\prime}(r)+\frac{1}{r}\right)\right\}=\kappa p_{t}(r),
\end{aligned}
$$

in which $\rho(r)$ is the energy density, ${ }^{2} p_{r}(r)$ is the radial pressure, and $p_{t}(r)$ is the lateral pressure. We can complete the EFE with the expression of the conservation of the stressenergy tensor, which can be written in the same orthonormal reference frame

$p_{r}^{\prime}(r)=\frac{2}{r}\left(p_{t}(r)-p_{r}(r)\right)-\left(\rho(r)+p_{r}(r)\right) \phi^{\prime}(r)$.

The purpose of this paper is to establish if the Casimir energy can be really considered as a source for a TW. In Ref. [12], this proposal was examined concluding that the plate separation should be smaller than the electron Compton wavelength; this means that the physical apparatus cannot be realized. Later Visser in his book [11] proposed a realistic model for the total stress-energy tensor (SET) represented by

\footnotetext{
${ }^{2}$ However, if $\rho(r)$ represents the mass density, then we have to replace $\rho(r)$ with $\rho(r) c^{2}$.
}

$$
\begin{aligned}
T_{\sigma}^{\mu \nu}= & \sigma \hat{t}^{\mu} \hat{t}^{\nu}[\delta(z)+\delta(z-a)] \\
& +\Theta(z) \Theta(a-z) \frac{\hbar c \pi^{2}}{720 a^{4}}\left[\eta^{\mu \nu}-4 \hat{z}^{\mu} \hat{z}^{\nu}\right],
\end{aligned}
$$

where $\hat{t}^{\mu}$ is a unit time-like vector, $\hat{z}^{\mu}$ is a normal vector to the plates and $\sigma$ is the mass density of the plates. He concluded that the mass of the plates compensates sufficiently much for the negative energy density, forbidding therefore the creation of a TW. Nonetheless, in Refs. [11-13], nothing has been said as regards the possible form of the shape function $b(r)$ and the related redshift function $\phi(r)$. Since an EoS is implicitly working, namely $p_{r}(r)=\omega \rho(r)$, with $\omega=3$, one is tempted to impose the simplest choice on the redshift function, namely $\phi^{\prime}(r)=0$. This assumption yields the following form for the metric:

$d s^{2}=-d t^{2}+\frac{d r^{2}}{1-\left(\frac{r_{0}}{r}\right)^{\frac{4}{3}}}+r^{2} d \Omega^{2}$

leading to the following SET:

$T_{\mu \nu}=\frac{1}{\kappa r^{2}}\left(\frac{r_{0}}{r}\right)^{\frac{\omega+1}{\omega}} \operatorname{diag}\left(-\frac{1}{\omega},-1, \frac{\omega+1}{\omega}, \frac{\omega+1}{\omega}\right)$,

which, for $\omega=3$, does not have any connection with the original Casimir SET. Thus it becomes important to explore other possibilities which can lead to the best correspondence between a TW and the Casimir SET. For instance, one can examine the consequences of the constraint imposed by a quantum weak energy condition (QWEC),

$\rho(r)+p_{r}(r)=-f(r) \quad f(r)>0 \quad \forall r \in\left[r_{0},+\infty\right)$.

The QWEC has been introduced for the first time in Ref. [18] and subsequently was used in cosmology in Ref. [17].

To the best of our knowledge, in the context of traversable wormholes the first appearance of the QWEC (14) is in Ref. [19], with

$f(r)=A\left(r_{0} / r\right)^{\alpha}$,

where $A$ is an appropriate constant introduced to describe an energy density. Note that the condition (14) has a direct connection with the volume integral quantifier, which provides information about the total amount of averaged null energy condition (ANEC) violating matter in the spacetime [21]. This is defined by

$I_{V}=\int\left[\rho(r)+p_{r}(r)\right] d V$, 
and for the line element (6), one can write

$I_{V}=\frac{1}{\kappa} \int(r-b(r))\left[\ln \left(\frac{e^{2 \phi(r)}}{1-\frac{b(r)}{r}}\right)\right]^{\prime} d r$,

where the measure $d V$ has been changed into $r^{2} d r$. For example, the calculation of $I_{V}$ for the traversable wormhole [10],

$d s^{2}=-d t^{2}+\frac{d r^{2}}{1-r_{0} / r}+r^{2} d \Omega^{2}$,

leads to

$$
\begin{aligned}
I_{V} & =-\frac{1}{\kappa} \int\left(r-r_{0}\right)\left[\ln \left(1-\frac{r_{0}}{r}\right)\right]^{\prime} d r \\
& =-\frac{r_{0}}{\kappa}[\ln (r)]_{r_{0}}^{+\infty} \rightarrow-\infty .
\end{aligned}
$$

This expression is diverging when $r \rightarrow \infty$, indicating that an infinite amount of exotic matter is required to maintain the wormhole. We may conclude that this result prohibits the existence of zero radial tides, namely $\phi(r)=0$ and zero density wormholes, namely $b(r)=$ constant, implying $\rho(r)=0$. The interesting feature of the QWEC (14) is that $b(r)$ can be determined exactly, not only for the form introduced in Ref. [19], but even for a generic $f(r)$. Indeed, with the help of Eqs. (7) and (8), we can write

$$
\frac{b^{\prime}(r)}{r}+\left[2\left(1-\frac{b(r)}{r}\right) \phi^{\prime}(r)-\frac{b(r)}{r^{2}}\right]=-\kappa r f(r)
$$

and, following Ref. [19], we introduce the auxiliary function $u(r)=1-b(r) / r$, leading to

$u^{\prime}(r)-2 u(r) \phi^{\prime}(r)=-\kappa r f(r)$.

This equation can easily be integrated and the form of the solution is

$b(r)=r\left[1-\kappa \exp (2 \phi(r)) \int_{r_{0}}^{r} \exp \left(-2 \phi\left(r^{\prime}\right)\right) f\left(r^{\prime}\right) r^{\prime} d r^{\prime}\right]$,

where the integration constant was fixed taking into account the throat condition. Therefore fixing the form of $\phi(r)$, one can determine $b(r)$. As an example, we briefly report the constant case examined in Ref. [19]. We find

$$
\begin{aligned}
b(r) & =r\left[1-\kappa \exp (2 \phi(r)) A \int_{r_{0}}^{r} \exp \left(-2 \phi\left(r^{\prime}\right)\right) r^{\prime} d r^{\prime}\right] \\
& =r\left(1-\frac{\kappa A}{2}\left(r^{2}-r_{0}^{2}\right)\right)
\end{aligned}
$$

where we have set $f(r)=A$ and $\phi(r)=0$. This last condition is also known as the zero tidal force (ZTF) condition. It is immediate to recognize that the previous shape function does not represent a traversable wormhole [19]. It is also possible to apply the reverse procedure, namely we fix the form of the shape function and we compute the redshift function and we obtain

$$
\begin{aligned}
\phi(r)= & \phi\left(r_{0}\right) \\
& +\frac{1}{2} \int_{r_{0}}^{r}\left[\frac{b(\bar{r})}{\bar{r}^{2}}-\frac{b^{\prime}(\bar{r})}{\bar{r}}-\kappa \bar{r} f(\bar{r})\right] \\
& \times\left(1-\frac{b(\bar{r})}{\bar{r}}\right)^{-1} d \bar{r} .
\end{aligned}
$$

The rest of the paper is structured as follows. In Sect. 2 we continue the investigation to determine if the Casimir energy density (4) can be considered as a source for a traversable wormhole. In Sect. 3 we explore some of the properties of the solution found previously; in Sect. 4 we use the QWEC to determine which kind of shape function we can obtain. In Sect. 5 we determine under which condition the QWEC will produce a traversable wormhole. We summarize and conclude in Sect. 6. Units in which $\hbar=c=k=1$ are used throughout the paper.

\section{The Casimir traversable wormhole}

In this section we assume that our exotic matter will be represented by the Casimir energy density (4). Following Ref. [12], we promote the constant plates separation $a$ to a radial coordinate $r$. Even if the authors of Ref. [12] assume a Casimir device made by spherical plates, we have to say that the SET form they use is the same as obtained with the flat plate assumption. Therefore the curvature of the plates introduces some modification which, in this first approximation, will be neglected. We have also to observe that the replacement of $a$ with $r$ could make the stress-energy tensor (11), with $\sigma=0$, potentially not conserved, as expected. Our strategy begins with the examination of Eq. (7) leading to the following form of the shape function:

$$
\begin{aligned}
b(r) & =r_{0}-\frac{\pi^{3}}{90}\left(\frac{\hbar G}{c^{3}}\right) \int_{r_{0}}^{r} \frac{d r^{\prime}}{r^{\prime 2}}=r_{0}+\frac{\pi^{3} l_{p}^{2}}{r 90}-\frac{\pi^{3} l_{p}^{2}}{r_{0} 90} \\
& =r_{0}-\frac{r_{1}^{2}}{r_{0}}+\frac{r_{1}^{2}}{r} ; \quad r_{1}^{2}=\frac{\pi^{3} l_{p}^{2}}{90}
\end{aligned}
$$

where the throat condition $b\left(r_{0}\right)=r_{0}$ has been imposed. To see if the Casimir energy really generates a TW, we need to study the redshift function which obeys Eq. (8). Plugging the shape function (25) into the radial pressure (8), we find 


$$
\begin{aligned}
& \frac{2}{r}\left(1-\frac{r_{0}}{r}+\frac{r_{1}^{2}}{r_{0} r}-\frac{r_{1}^{2}}{r^{2}}\right) \phi^{\prime}(r) \\
& -\frac{r_{0}}{r^{3}}+\frac{r_{1}^{2}}{r_{0} r^{3}}-\frac{(1-\omega) r_{1}^{2}}{r^{4}}=0,
\end{aligned}
$$

where we have used the $\operatorname{EoS} p_{r}(r)=\omega \rho(r)$ and Eq. (7). The solution for a generic $\omega$ is

$$
\begin{aligned}
\phi(r)= & -\frac{1}{2}\left[\left(\omega r_{0}^{2}-r_{1}^{2}\right) \frac{\ln \left(r_{0} r+r_{1}^{2}\right)}{r_{0}^{2}+r_{1}^{2}}+(1-\omega) \ln (r)\right. \\
& \left.+\left(\omega r_{1}^{2}-r_{0}^{2}\right) \frac{\ln \left(r-r_{0}\right) \omega r_{1}^{2}}{r_{0}^{2}+r_{1}^{2}}\right]+\phi\left(r_{0}\right) .
\end{aligned}
$$

Since we have some freedom in fixing $\omega$ and $r_{0}$, we observe that when $\omega r_{1}^{2}-r_{0}^{2}>0$, we find a black hole. Basically, this is due to the presence of the term $\ln \left(r-r_{0}\right)$. When we fix ${ }^{3}$

$\omega r_{1}^{2}-r_{0}^{2}=0 \Longrightarrow \omega=\omega_{0}=\frac{r_{0}^{2}}{r_{1}^{2}}$,

we obtain a TW. Assuming the last choice, we can write

$\phi(r)=\frac{1}{2}(\omega-1) \ln \left(\frac{r(\omega+1)}{\omega r+r_{0}}\right)+\phi\left(r_{0}\right)$,

and for $r \rightarrow \infty$,

$\phi(r) \rightarrow \frac{1}{2}(\omega-1) \ln \left(\frac{\omega+1}{\omega}\right)+\phi\left(r_{0}\right)$,

therefore, in order to obtain the appropriate asymptotic flat limit, it is convenient to $\mathrm{fix}^{4}$

$\phi\left(r_{0}\right)=-\frac{1}{2}(\omega-1) \ln \left(\frac{\omega+1}{\omega}\right)$.

Then the redshift function reduces to

$\phi(r)=\frac{1}{2}(\omega-1) \ln \left(\frac{r \omega}{\omega r+r_{0}}\right)$

and the shape function becomes

$b(r)=\left(1-\frac{1}{\omega}\right) r_{0}+\frac{r_{0}^{2}}{\omega r}$.

$\overline{3 \text { When } \omega r_{1}^{2}-} r_{0}^{2}<0$, we obtain a singularity. See Appendix A for details.

${ }^{4}$ By imposing $\phi\left(r_{0}\right)=0$, the redshift function should be

$\phi(r)=\frac{1}{2}(\omega-1) \ln \left(\frac{r(\omega+1)}{\left(\omega r+r_{0}\right)}\right)$.
The last component of the SET we have to compute is $p_{t}$. With the help of Eq. (9), we find

$p_{t}(r)=\omega_{t}(r)\left(\frac{r_{0}^{2}}{\kappa \omega r^{4}}\right)=\omega_{t}(r) \rho(r)$,

where we have introduced an inhomogeneous EoS on the transverse pressure with

$\omega_{t}(r)=-\frac{\omega^{2}\left(4 r-r_{0}\right)+r_{0}(4 \omega+1)}{4\left(\omega r+r_{0}\right)}$,

and the final form of the SET is

$$
\begin{aligned}
T_{\mu \nu}= & \frac{r_{0}^{2}}{\kappa \omega r^{4}}[\operatorname{diag}(-1,-\omega, 1,1) \\
& \left.+\left(\omega_{t}(r)-1\right) \operatorname{diag}(0,0,1,1)\right]
\end{aligned}
$$

The conservation of the SET is satisfied but a comparison with the Casimir SET shows that there is an extra contribution on the transverse pressure: this is the consequence of having a function of the radial coordinate in front of the SET instead of a constant. It is interesting to observe that the first tensor is the Casimir SET iff $\omega=3$. Moreover, when

$\frac{r_{0}^{2}}{\kappa \omega}=\frac{r_{1}^{2}}{\kappa}=\frac{c^{4}}{8 \pi G} \frac{\pi^{3}}{90}\left(\frac{\hbar G}{c^{3}}\right)=\frac{\hbar c \pi^{2}}{720}$,

the identification is complete. However, this is true only for the expressions of $p_{r}(r)$ and $\rho(r)$. The extra contribution on $p_{t}(r)$ tells us that the real source is a variant of the Casimir SET. Combining the redshift function (33) with the shape function (34), we can write the line element (6) in the following way:

$$
\begin{aligned}
d s^{2}= & -\left(\frac{r \omega}{\left(\omega r+r_{0}\right)}\right)^{\omega-1} d t^{2} \\
& +\frac{d r^{2}}{1-\frac{1}{r}\left(\left(1-\frac{1}{\omega}\right) r_{0}+\frac{r_{0}^{2}}{\omega r}\right)}+r^{2} d \Omega^{2}
\end{aligned}
$$

and for the special value $\omega=3$, we find

$\phi(r)=\ln \left(\frac{3 r}{3 r+r_{0}}\right) \quad$ and $\quad b(r)=\frac{2 r_{0}}{3}+\frac{r_{0}^{2}}{3 r}$

whose line element is

$d s^{2}=-\left(\frac{3 r}{3 r+r_{0}}\right)^{2} d t^{2}+\frac{d r^{2}}{1-\frac{2 r_{0}}{3 r}-\frac{r_{0}^{2}}{3 r^{2}}}+r^{2} d \Omega^{2}$ 
describing a TW of Planckian size. ${ }^{5}$ The corresponding SET (37) becomes

$$
\begin{aligned}
T_{\mu \nu}= & \frac{r_{0}^{2}}{3 \kappa r^{4}}[\operatorname{diag}(-1,-3,1,1) \\
& \left.+\left(\frac{6 r}{3 r+r_{0}}\right) \operatorname{diag}(0,0,1,1)\right] .
\end{aligned}
$$

Note that the inhomogeneous function (36) is such that

$$
r \in\left[r_{0},+\infty\right) \Longrightarrow \omega_{t}(r) \in\left[-\frac{5}{2},-3\right)
$$

and the transverse pressure on the throat becomes

$p_{t}\left(r_{0}\right)=\frac{5}{6 \kappa r_{0}^{2}}$.

It is interesting also to observe that if $\omega=1$, we obtain the Ellis-Bronnikov (EB) wormhole of sub-Planckian $\operatorname{size}^{6}[30$, 31]. Even in this case, the SET is conserved, but to establish a connection with the Casimir SET, we need to write the SET of (37) in the following way ${ }^{7}$ :

$$
\begin{aligned}
T_{\mu \nu} & =\frac{r_{0}^{2}}{\kappa r^{4}}[\operatorname{diag}(-1,-1,1,1)] \\
& =\frac{r_{0}^{2}}{2 \kappa r^{4}}[\operatorname{diag}(-1,-3,1,1)+\operatorname{diag}(-1,1,1,1)] .
\end{aligned}
$$

To complete the discussion is also useful to include the form for the Kretschmann scalar, which, for $\omega=1$, assumes the form

$R_{\alpha \beta \gamma \delta} R^{\alpha \beta \gamma \delta}=\frac{12 r_{0}^{4}}{r^{8}}$

while, for $\omega=3$, it becomes

$$
\begin{aligned}
R_{\alpha \beta \gamma \delta} R^{\alpha \beta \gamma \delta}= & \frac{r_{0}^{2}}{9\left(6 r+r_{0}\right)^{2}\left(3 r+r r_{0}\right)^{2} r^{8}} \\
\times & {\left[9720 r^{6}+14256 r^{5} r_{0}\right.} \\
& +18252 r^{4} r_{0}^{2}+8856 r^{3} r_{0}^{3} \\
& \left.+2112 r^{2} r_{0}^{4}+248 r r_{0}^{5}+12 r_{0}^{6}\right] .
\end{aligned}
$$

The general expression for a generic $\omega$ is quite lengthy and it is not particularly illuminating. In the next section, we are going to explore some of the features of the line element (41).

\footnotetext{
$\overline{5 \text { We have } r_{0}}=\sqrt{3} r_{1} \simeq 1.0166 l_{P}$.

${ }^{6} r_{0}=\sqrt{\frac{\pi^{3}}{90}} l_{p}=0.58695 l_{p}$.

7 The SET is represented in an orthonormal frame.
}

\section{Properties of the Casimir wormhole}

In Sect. 2, we have introduced the shape function (41) obtained by the Casimir energy. Here we want to discuss some of its properties, even if the wormhole has Planckian size. The first quantity we are going to analyze is the proper radial distance which is related to the shape function by

$$
\begin{aligned}
l(r)= & \pm \int_{r_{0}}^{r} \frac{d r^{\prime}}{\sqrt{1-\frac{b\left(r^{\prime}\right)}{r^{\prime}}}}= \pm \int_{r_{0}}^{r} \frac{d r^{\prime}}{\sqrt{1-\frac{2 r_{0}}{3 r^{\prime}}-\frac{r_{0}^{2}}{3 r^{\prime 2}}}} \\
= & \pm \frac{\sqrt{3} \sqrt{\left(3 r+r_{0}\right)\left(r-r_{0}\right)}}{3} \\
& +\frac{r_{0}}{3} \ln \left(\frac{3 r-r_{0}+\sqrt{3} \sqrt{\left(3 r+r_{0}\right)\left(r-r_{0}\right)}}{2 r_{0}}\right) .
\end{aligned}
$$

We find

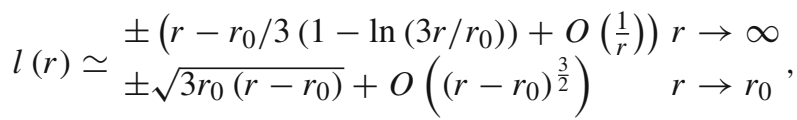

where the " \pm " depends on the wormhole side we are. The proper radial distance is an essential tool to estimate the possible time trip in going from one station located in the lower universe, say at $l=-l_{1}$, and ending up in the upper universe station, say at $l=l_{2}$. Following Ref. [10], we shall locate $l_{1}$ and $l_{2}$ at a value of the radius such that $l_{1} \simeq l_{2} \simeq 10^{4} r_{0}$ that it means $1-b(r) / r \simeq 1$. Assume that the traveler has a radial velocity $v(r)$, as measured by a static observer positioned at $r$. One may relate the proper distance traveled $d l$, the radius traveled $d r$, the coordinate time lapse $d t$, and the proper time lapse as measured by the observer $d \tau$, by the following relationships:

$$
v=e^{-\phi(r)} \frac{d l}{d t}=e^{-\phi(r)}\left(1-\frac{b(r)}{r}\right)^{-\frac{1}{2}} \frac{d r}{d t}
$$

and

$$
\begin{aligned}
& v \gamma=\frac{d l}{d \tau}=\mp\left(1-\frac{b(r)}{r}\right)^{-\frac{1}{2}} \frac{d r}{d \tau} ; \\
& \gamma=\left(1-\frac{v^{2}(r)}{c^{2}}\right)^{-\frac{1}{2}},
\end{aligned}
$$

respectively. If the traveler journeys with constant speed $v$, then the total time is given by

$$
\begin{aligned}
\Delta t= & \int_{r_{0}}^{r} \frac{e^{-\phi\left(r^{\prime}\right)} d r^{\prime}}{v \sqrt{1-\frac{b\left(r^{\prime}\right)}{r^{\prime}}}}=\frac{1}{4 v} \sqrt{9 r^{2}-6 r_{0} r-3 r_{0}^{2}} \\
& +\frac{r_{0}}{2 v} \ln \left(\frac{3 r}{2 r_{0}}+\frac{\sqrt{9 r^{2}-6 r r_{0}-3 r_{0}^{2}}}{2 r_{0}}-\frac{1}{2}\right),
\end{aligned}
$$




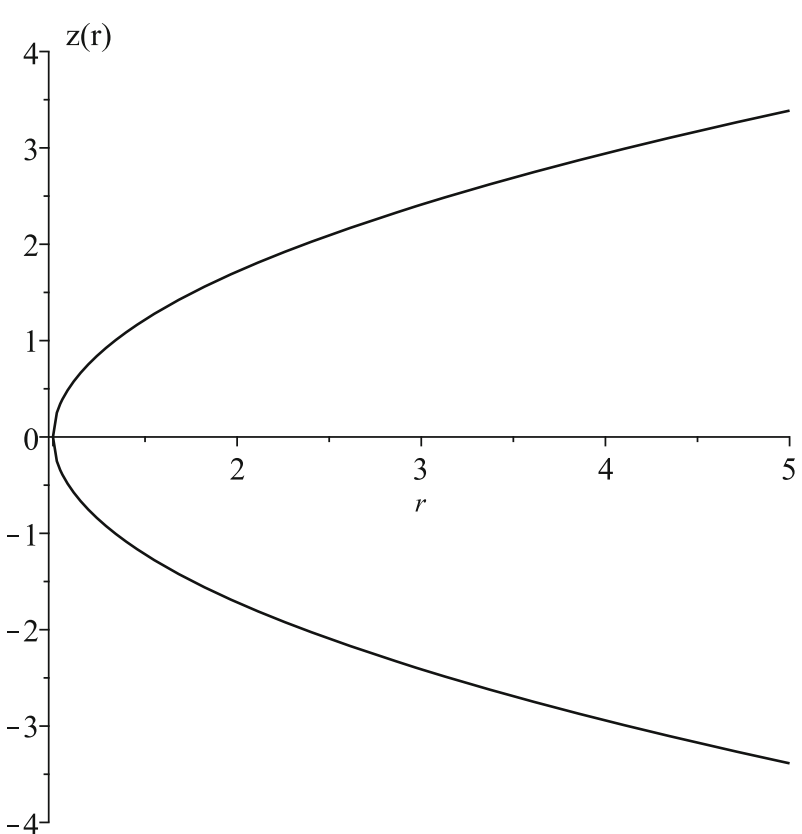

Fig. 1 Representation of the embedding diagram

while the proper total time is

$$
\begin{aligned}
\Delta \tau= & \int_{r_{0}}^{r} \frac{d r^{\prime}}{v \sqrt{1-\frac{b\left(r^{\prime}\right)}{r^{\prime}}}}=\frac{1}{3 v} \sqrt{9 r^{2}-6 r r_{0}-3 r_{0}^{2}} \\
& +\frac{r_{0}}{3 v} \ln \left(\frac{3 r}{2 r_{0}}+\frac{\sqrt{9 r^{2}-6 r r_{0}-3 r_{0}^{2}}}{2 r_{0}}-\frac{1}{2}\right) .
\end{aligned}
$$

As we can see $\Delta \tau \simeq \Delta t$. On the same ground, we can compute the embedded surface, which is defined by (Fig. 1)

$z(r)= \pm \int_{r_{0}}^{r} \frac{d r^{\prime}}{\sqrt{\frac{b\left(r^{\prime}\right)}{r^{\prime}}-1}}$

and, in the present case, we find

$$
\begin{aligned}
z(r)= & \pm \sqrt{\frac{r_{0}}{3}} \int_{r_{0}}^{r} \frac{\sqrt{2 r^{\prime}+r_{0}} d r^{\prime}}{\sqrt{\left(r^{\prime}-r_{0}\right)\left(r^{\prime}+r_{0} / 3\right)}} \\
= & \frac{2 r_{0}}{9}\left[F\left(\sqrt{3} \sqrt{\frac{r-r_{0}}{r_{0}+3 r}}, \frac{1}{3}\right)\right. \\
& \left.+8 \Pi\left(\sqrt{3} \sqrt{\frac{r-r_{0}}{r_{0}+3 r}}, 1, \frac{1}{3}\right)\right],
\end{aligned}
$$

where $F(\varphi, k)$ is the elliptic integral of the first kind and $\Pi(\varphi, n, k)$ is the elliptic integral of the third kind. To further investigate the properties of the shape function (41), we consider the computation of the total gravitational energy for a wormhole [33], defined as

$E_{G}(r)=\int_{r_{0}}^{r}\left[1-\sqrt{\frac{1}{1-b\left(r^{\prime}\right) / r^{\prime}}}\right] \rho\left(r^{\prime}\right) d r^{\prime} r^{\prime 2}$

$$
+\frac{r_{0}}{2 G}=M-M_{ \pm}^{P},
$$

where $M$ is the total mass $M$ and $M^{P}$ is the proper mass, respectively. Even in this case, the " \pm " depends on the wormhole side we are. In particular

$$
\begin{aligned}
M & =\int_{r_{0}}^{r} 4 \pi \rho\left(r^{\prime}\right) r^{\prime 2} d r^{\prime}+\frac{r_{0}}{2 G} \\
& =\frac{1}{2 G}\left(\frac{2 r_{0}}{3}+\frac{r_{0}^{2}}{3 r}-r_{0}\right)+\frac{r_{0}}{2 G} \\
& =\frac{1}{2 G}\left(\frac{2 r_{0}}{3}+\frac{r_{0}^{2}}{3 r}\right) \underset{r \rightarrow \infty}{\simeq} \frac{r_{0}}{3 G}
\end{aligned}
$$

and

$$
\begin{aligned}
M_{ \pm}^{P} & = \pm \int_{r_{0}}^{r} \frac{4 \pi \rho\left(r^{\prime}\right) r^{\prime 2}}{\sqrt{1-b\left(r^{\prime}\right) / r^{\prime}}} d r^{\prime} \\
& =\mp \frac{\sqrt{3} c^{4} r_{0}^{2}}{6 G} \int_{r_{0}}^{r} \frac{d r^{\prime}}{r^{\prime} \sqrt{3 r^{\prime 2}-6 r_{0} r^{\prime}-r_{0}^{2}}} \\
& =\mp \frac{\sqrt{3} c^{4} r_{0}}{12 G}\left(\pi-2 \arctan \left(\frac{r_{0}+r}{\sqrt{3 r^{2}-2 r_{0} r-r_{0}^{2}}}\right)\right) \\
& \underset{r \rightarrow \infty}{\simeq} \mp \frac{\sqrt{3} \pi c^{4} r_{0}}{18 G} .
\end{aligned}
$$

An important traversability condition is that the acceleration felt by the traveler should not exceed Earth's gravity, $g_{\oplus} \simeq$ $980 \mathrm{~cm} / \mathrm{s}^{2}$. In an orthonormal basis of the traveler's proper reference frame, we can find

$$
|\mathbf{a}|=\left|\sqrt{1-\frac{b(r)}{r}} e^{-\phi(r)}\left(\gamma e^{\phi(r)}\right)^{\prime}\right| \leq \frac{g_{\oplus}}{c^{2}} .
$$

If we assume a constant speed and $\gamma \simeq 1$, then we can write

$$
|\mathbf{a}|=\left|\sqrt{1-\frac{2 r_{0}}{3 r}-\frac{r_{0}^{2}}{3 r}} \frac{r_{0}}{r\left(3 r+r_{0}\right)}\right| \leq \frac{g_{\oplus}}{c^{2}} .
$$

We can see that, in proximity of the throat, the traveler has no acceleration. Always following Ref. [10], we can estimate the tidal forces by imposing an upper bound represented by $g_{\oplus}$. The radial tidal constraint,

$$
\begin{aligned}
& \mid\left(1-\frac{b(r)}{r}\right)\left[\phi^{\prime \prime}(r)+\left(\phi^{\prime}(r)\right)^{2}\right. \\
& \left.\quad-\frac{b^{\prime}(r) r-b(r)}{2 r(r-b(r))} \phi^{\prime}(r)\right] c^{2}|| \eta^{\hat{1}^{\prime}} \mid \leq g_{\oplus},
\end{aligned}
$$

constrains the redshift function, and the lateral tidal constraint,

$$
\mid \frac{\gamma^{2} c^{2}}{2 r^{2}}\left[\frac{v^{2}(r)}{c^{2}}\left(b^{\prime}(r)-\frac{b(r)}{r}\right)\right.
$$




$$
\left.+2 r(r-b(r)) \phi^{\prime}(r)\right]|| \eta^{\hat{2}^{\prime}} \mid \leq g_{\oplus},
$$

constrains the velocity with which observers traverse the wormhole. $\eta^{\hat{1}^{\prime}}$ and $\eta^{\hat{2}^{\prime}}$ represent the size of the traveler. In Ref. [10], they are fixed approximately at equal values, at the symbolic value of $2 \mathrm{~m}$. Close to the throat, the radial tidal constraint (61) becomes ${ }^{8}$

$$
\begin{gathered}
\left|\left[\frac{b(r)-b^{\prime}(r) r}{2 r^{2}} \phi^{\prime}(r)\right]\right| \leq \frac{g_{\oplus}}{c^{2}\left|\eta^{\hat{\prime}^{\prime}}\right|} \simeq\left(10^{8} m\right)^{-2} \\
\Longrightarrow \quad 10^{8} m \lesssim r_{0} .
\end{gathered}
$$

For the lateral tidal constraint, we find

$$
\begin{aligned}
& \frac{v^{2} r_{0}}{3 r^{4}}\left(r+r_{0}\right)\left|\eta^{\hat{2}^{\prime}}\right| \lesssim g_{\oplus} \Longrightarrow v \lesssim r_{0} \sqrt{\frac{3 g_{\oplus}}{4}} \\
& \Longrightarrow \quad v \lesssim 2.7 r_{0} \mathrm{~m} / \mathrm{s} .
\end{aligned}
$$

If the observer has a vanishing $v$, then the tidal forces are null. We can use these last estimates to complete the evaluation of the crossing time which approximately is

$\Delta t \simeq 2 \times 10^{4} \frac{3 r_{0}}{4 v} \simeq 5 \times 10^{3} s$,

which is in agreement with the estimates found in Ref. [10]. The last property we are going to discuss is the "total amount" of ANEC violating matter in the spacetime [21] which is described by Eq. (17). For the metric (41), one obtains

$I_{V}=-\frac{1}{\kappa} \int_{r_{0}}^{\infty} \frac{4 r_{0}^{2}}{3 r^{2}} d r=-\frac{4 r_{0}}{3 \kappa}$.

Differently from what we have computed in Eq. (19), this time the result is finite everywhere. Therefore we can conclude that, in proximity of the throat, the ANEC can be arbitrarily small. In the next section, we will explore the consequences of assuming a QWEC to see its relationship with the Casimir energy and its connection with the formation of a TW.

\section{Global monopoles from QWEC}

As pointed out in [18], the Casimir energy and the QWEC have a strong connection. Therefore, it appears interesting which kind of shape function we can extract by imposing such a profile on the relationship leading to Eq. (22). By assuming

\footnotetext{
${ }^{8}$ Note that if we put the Planckian value in $r_{0}$ obtained in (40), then one finds $\left|\eta^{\hat{1}^{\prime}}\right| \leq 1.0573 \times 10^{-86} \mathrm{~m}$. This means that with a Planckian wormhole nothing can traverse it.
}

a vanishing redshift function, we can write

$b(r)=r\left[1-\kappa \int_{r_{0}}^{r} f\left(r^{\prime}\right) r^{\prime} d r^{\prime}\right]$

and with the help of (15) one gets

$$
\begin{aligned}
b(r) & =r\left[1-\kappa A r_{0}^{\alpha} \int_{r_{0}}^{r} \frac{d r^{\prime}}{r^{\prime \alpha-1}}\right] \\
& =r\left[\left(1-\frac{\kappa A r_{0}^{\alpha}}{\alpha-2} \frac{1}{r_{0}^{\alpha-2}}\right)+\frac{\kappa A r_{0}^{\alpha}}{\alpha-2} \frac{1}{r^{\alpha-2}}\right], \quad \alpha \neq 2,
\end{aligned}
$$

while, for $\alpha=2$, one finds

$b(r)=r\left[1-\kappa A r_{0}^{2} \int_{r_{0}}^{r} \frac{d r^{\prime}}{r^{\prime}}\right]=r\left[1-\kappa A r_{0}^{2} \ln \left(\frac{r}{r_{0}}\right)\right]$.

The shape functions (68) and (69) have been analyzed in Ref. [19] and we know that they will produce a global monopole [20]. Nevertheless, an interesting shape function can be obtained if one plugs the redshift function (40) into Eq. (22). Indeed, one finds

$$
\begin{aligned}
& b(r)=r \\
& \quad \times\left(1-\frac{\kappa r^{2} A\left(2\left(8 \alpha^{2}-12 \alpha+1\right) r_{0}^{2}-c(r)\left(r_{0} / r\right)^{\alpha}\right)}{\left(3 r+r_{0}\right)^{2} \alpha(\alpha-1)(\alpha-2)}\right),
\end{aligned}
$$

where

$c(r)=\left(3 r+r_{0}\right)^{2} \alpha(\alpha-1)-2\left(r_{0}^{2}+3 r r_{0}\right) \alpha+2 r_{0}^{2}$.

When $r \rightarrow \infty$, we have the following asymptotic behavior for $\alpha>2$ :

$b(r) \simeq r\left(1-\frac{2 A \kappa r_{0}^{2}\left(8 \alpha^{2}-12 \alpha+1\right)}{9 \alpha(\alpha-1)(\alpha-2)}\right)$,

which implies that, even in this case, we have a global monopole. Indeed, plugging the shape function (51) into the original metric (6), we can write

$$
\begin{aligned}
d s^{2}= & -\left(\frac{4 r}{3 r+r_{0}}\right)^{2} d t^{2} \\
& +\frac{\left(3 r+r_{0}\right)^{2} \alpha(\alpha-1)(\alpha-2) d r^{2}}{\kappa r^{2} A\left(2\left(8 \alpha^{2}-12 \alpha+1\right) r_{0}^{2}-c(r)\left(r_{0} / r\right)^{\alpha}\right)} \\
& +r^{2} d \Omega^{2} \\
& \underset{r \rightarrow \infty}{\simeq}-\frac{16}{9} d t^{2}+\frac{9 \alpha(\alpha-1)(\alpha-2)}{2 A \kappa r_{0}^{2}\left(8 \alpha^{2}-12 \alpha+1\right)} d r^{2}+r^{2} d \Omega^{2} \\
= & \underset{r \rightarrow \infty}{\simeq}-\frac{16}{9} d t^{2}+d \tilde{r}^{2}+\Delta \tilde{r}^{2} d \Omega^{2}
\end{aligned}
$$


where we have rescaled the radial coordinate and where we have defined

$\Delta=\frac{2 A \kappa r_{0}^{2}\left(8 \alpha^{2}-12 \alpha+1\right)}{9 \alpha(\alpha-1)(\alpha-2)}$.

If $\Delta>0$ we have an excess of the solid angle and this happens when

$\alpha>2 ; \quad 0<\alpha<(3-\sqrt{7}) / 4 ; \quad 1<\alpha<(3+\sqrt{7}) / 4$.

On the other hand when

$\alpha<0 ; \quad(3-\sqrt{7}) / 4<\alpha<1 ; \quad(3+\sqrt{7}) / 4<\alpha<2$

we have a deficit of the solid angle, namely $\Delta<0$. The authors of Ref. [19] interpret the metric (68) as a wormhole carrying a global monopole: the same interpretation can be applied to the metric (73). For completeness, we can compute the SET components like the energy density,

$\rho(r)=\frac{1}{\kappa r^{2}}\left\{1-\frac{A \kappa\left(\rho^{1}(r)\left(r_{0} / r\right)^{\alpha}+\rho^{2}(r)\right)}{9 \alpha(\alpha-1)(\alpha-2)\left(3 r+r_{0}\right)^{2}}\right\}$,

where

$$
\begin{aligned}
\rho^{1}(r)= & {\left[6 r^{2}(\alpha-2) \alpha(6 r+\alpha-4) r r_{0}\right.} \\
& -9 r^{2}(\alpha+6 r-5)(\alpha-1) \alpha \\
& \left.-(6 r+\alpha-3)(\alpha-1)(\alpha-2) r_{0}^{2}\right], \\
\rho^{2}(r)= & 6(2 r-1)\left(8 \alpha^{2}-12 \alpha+1\right) r_{0}^{2} .
\end{aligned}
$$

The radial pressure is simply

$p_{r}(r)=-\frac{r}{\kappa r^{2}}\left(1-\frac{c(r)}{\left(3 r+r_{0}\right)^{2} \alpha\left(\alpha^{2}-3 \alpha+2\right)}\right)$

and finally the transverse pressure is

$p_{t}(r)=\frac{9 \kappa A\left[p_{t}^{1}(r)+p_{t}^{2}(r)+p_{t}^{3}(r)+4 r_{0}^{4}\left(8 \alpha^{2}-12 \alpha+1\right) / 3\right]}{2(\alpha-1)(\alpha-2) \alpha\left(3 r+r_{0}\right)^{4}}$,

where we have defined

$$
\begin{aligned}
p_{t}^{1}(r)= & 6(\alpha-2) \alpha r_{0} r\left(\left(\frac{2 \alpha-8}{9}\right) r_{0}^{2}\right. \\
& \left.+\left(r r_{0}+r^{2}\right)(\alpha-1)\right)\left(\frac{r+0}{r}\right)^{2} \\
p_{t}^{2}(r)= & 9 \alpha r^{2}\left(\left(\frac{2 \alpha-10}{9}\right) r_{0}^{2}\right.
\end{aligned}
$$

$$
\begin{aligned}
& \left.+\left(r r_{0}+r^{2}\right)(\alpha-2)\right)(\alpha-1)\left(\frac{r_{0}}{r}\right)^{\alpha}, \\
p_{t}^{3}(r)= & \left(+\alpha r r_{0}+\alpha r^{2}\left(\frac{r_{0}}{r}\right)^{\alpha}\right. \\
& \left.+\alpha r r_{0}+\alpha r^{2}\right)\left(\frac{r_{0}}{r}\right)^{\alpha} .
\end{aligned}
$$

On the throat we find

$$
\rho\left(r_{0}\right)=\frac{1-A \kappa r_{0}^{2}}{\kappa r_{0}^{2}}>0 \Longleftrightarrow 1>A \kappa r_{0}^{2},
$$

$p_{r}\left(r_{0}\right)=-\frac{1}{\kappa r_{0}^{2}}<0, \quad p_{t}\left(r_{0}\right)=\frac{5 A}{8}>0$.

Once again, for the special case $\alpha=4$, one gets

$$
\begin{aligned}
\rho(r) & =\frac{\left(-3 A \kappa r_{0}^{2}+4\right) r^{2}-A \kappa r_{0}^{4}}{4 \kappa r^{4}}, \\
p_{r}(r) & =\frac{3 \kappa r^{2} A r_{0}^{2}-2 A \kappa r_{0}^{3} r-A \kappa r_{0}^{4}-4 r^{2}}{4 \kappa r^{2}}, \\
p_{t}(r) & =\frac{\left(9 r+r_{0}\right) r_{0}^{4} A}{4 r^{4}\left(3 r+r_{0}\right)} .
\end{aligned}
$$

\section{The return of the traversable wormhole and the disappearance of the global monopole}

The throat condition constrains the shape function to have a fixed point, but not its value. Thanks to this freedom, we can reexamine the shape functions obtained previously and we show that, for appropriate choices of the parameters, a TW without a monopole can appear. For this purpose, let us begin from Eq. (68) and by observing that nothing forbids the following identification:

$1-\frac{\kappa A r_{0}^{\alpha}}{\alpha-2} \frac{1}{r_{0}^{\alpha-2}}=0 \Longleftrightarrow r_{0}=\sqrt{\frac{\alpha-2}{\kappa A}}$,

and one obtains the following relationship:

$b(r)=\frac{r_{0}^{\alpha-2}}{r^{\alpha-3}}, \quad \alpha \neq 2$.

Note that the assumption (89) is not possible when $\alpha=2$. Since the shape function (90) has been obtained imposing ZTF, the computation of the SET is immediate and we get

$$
\begin{aligned}
& \rho(r)=-(\alpha-3) \frac{r_{0}^{\alpha-2}}{\kappa r^{\alpha}}, \quad p_{r}(r)=-\frac{r_{0}^{\alpha-2}}{\kappa r^{\alpha}}, \\
& p_{t}(r)=(\alpha-2) \frac{r_{0}^{\alpha-2}}{2 \kappa r^{\alpha}} .
\end{aligned}
$$

For the particular case of $\alpha=4$, we recover the EB wormhole and a direct comparison with the Casimir SET allows one to fix also the constant $A$. Indeed, one finds 


$$
\begin{aligned}
\rho(r) & =-\frac{r_{0}^{2}}{\kappa r^{4}}=-\frac{\hbar c \pi^{2}}{720 r^{4}} \\
& \Longrightarrow r_{0}^{2}=\frac{\pi^{3} l_{P}^{2}}{90} \\
& \Longrightarrow A=\frac{45 \hbar c}{2 l_{P}^{4} \pi^{4}} .
\end{aligned}
$$

However, it is immediate to recognize that the energy density $A$ is so huge that it cannot have a physical meaning. Moreover, we can also impose

$A=-\frac{4 \hbar c \pi^{2}}{720 a^{4}} \Longrightarrow a=0.7 l_{P}$

where $a$ is the fixed plate distance. This further constraint is even worse than that found in Ref. [12]. Nevertheless if we constrain only the relationship (89),

$r_{0}=\sqrt{\frac{\alpha-2}{\kappa A}}=\frac{a^{2}}{l_{P}} \sqrt{\frac{90(\alpha-2)}{4 \pi^{3}}}$,

and we consider a standard Casimir separation with $a \simeq$ $1 \mu \mathrm{m}$ and $\alpha=4$, we find an opposite huge result

$r_{0} \simeq 3.7273 \times 10^{22} \mathrm{~m}$

We need to reach a plate separation of the order of the $f m$, to have a throat of the order of $10^{5} \mathrm{~m}$. One could be tempted to use the arbitrariness of $\alpha$. However, $\alpha>3$, otherwise the energy density becomes positive. The other case where the TW returns and the global monopole disappears is represented by the shape function (51), whose asymptotic behavior is represented by Eq. (72). With the assumption

$A=\frac{9 \alpha(\alpha-2)(\alpha-1)}{2 \kappa r_{0}^{2}\left(8 \alpha^{2}-12 \alpha+1\right)}$

one finds the TW shape function $\forall \alpha>2$

$b(r)=r\left(1-\frac{9 r^{2}\left(2\left(8 \alpha^{2}-12 \alpha+1\right) r_{0}^{2}-c(r)\left(r_{0} / r\right)^{\alpha}\right)}{2 r_{0}^{2}\left(8 \alpha^{2}-12 \alpha+1\right)\left(3 r+r_{0}\right)^{2}}\right)$.

In particular for $\alpha=4$, one finds

$$
\begin{aligned}
b(r) & =\frac{r_{0}}{3\left(3 r+r_{0}\right)^{2} r}\left[\left(2 r+r_{0}\right)\left(3 r+r_{0}\right)^{2}\right] \\
& =\frac{2 r_{0}}{3}+\frac{r_{0}^{2}}{3 r}
\end{aligned}
$$

in agreement with the shape function described in (40). The SET coincides with the one described by (42). In particular one finds

$$
\begin{aligned}
& \rho(r)=-\frac{\pi^{2} \hbar c}{720 r^{4}}, \quad p_{r}(r)=-\frac{3 \pi^{2} \hbar c}{720 r^{4}} \\
& p_{t}(r)=\frac{\pi^{2} \hbar c}{720 r^{4}}\left(\frac{9 r+r_{0}}{3 r+r_{0}}\right),
\end{aligned}
$$

where we have fine-tuned the parameters in such a way as to produce the correct values of the Casimir energy density and pressure. As one can see, the disagreement with the transverse pressure persists. If we put in the physical numbers we have used for the (90) case, we find no substantial difference with that case.

\section{Conclusions}

In this paper, we have extended the study began by Morris, Thorne and Yurtsever in Ref. [12] and subsequently explored by Visser [11] on the Casimir effect as a possible source for a TW. By imposing an EoS, we have discovered a solution depending on the ratio between the throat radius and $r_{1}$ which is directly connected with the Planck length. As shown in Eq. (28) if $\omega>\omega_{0}$, we have a black hole, while if $\omega=\omega_{0}$, we have a TW. Note also that, for $\omega<\omega_{0}$, we have a singularity as shown in Appendix A. For the TW case, one finds that, for the special value $\omega=3$, the size of the TW is Planckian and therefore it is traversable in principle but not in practice. The TW (39) reproduces the original Casimir energy density (4) and pressure (3), but the transverse pressure is in disagreement with the one associated with the TW (39). This is a consequence of us having assumed a variable separation between the plates instead of a constant separation like for the original Casimir effect. Therefore the correspondence between the original Casimir SET and the one computed by means of the line element (39) is not one to one. Nevertheless, it is remarkable that a solution connecting the Casimir energy and a TW has been found because, to the best of our knowledge, in the literature nothing is said about this point. It is also remarkable that the same EoS with $\omega=1$ is able to reproduce the EB wormhole, but even in this case the correspondence with the Casimir SET is not one to one. For this reason we have explored the possibility of supporting the TW (39) with other forms of constraint. An interesting condition comes from the QWEC which, in general, produces a global monopole. However, even in this case it is always possible to use the arbitrariness of the throat and eliminate the global monopole in favor of an appearing TW. Unfortunately, even if the QWEC corroborates the results obtained in Sect. 2, when one puts numbers inside the parameters, the expectations are too far out to be momentarily interesting. A comment about the EoS with $\omega=3$ is in order. Since it is the NEC that must be violated, many proposals to keep $\rho(r)>0$ have been made [22-24]. However, even if the results are encouraging, we have to say that there is no knowledge on how to 
build "phantom energy" in practice. I recall that the phantom energy obeys the following relationship:

$$
\begin{aligned}
p_{r}(r) & =\omega \rho(r), \Longrightarrow p_{r}(r)+\rho(r)<0 \\
& \Longleftrightarrow(1+\omega) \rho(r)<0,
\end{aligned}
$$

which implies

$\rho(r)>0, \Longrightarrow \omega<-1$.

On this ground the Casimir effect offers a viable interesting realizable model of exotic matter which, however, can contribute only at the Planck scale. Always on the side of phantom energy we proposed the idea of self-sustained traversable wormholes, namely TW sustained by their own quantum fluctuations [25-29]. Even in this case, because the quantum fluctuation carried by the graviton behaves like the ordinary Casimir effect, we found there to be no need for a phantom contribution. In this context, in a next paper we will explore how a system behaves formed by the Casimir $T W$, here analyzed, and the corresponding self-sustained TW version.

Acknowledgements I would like to thank Francisco S.N. Lobo for useful discussions and suggestions for the traversable wormhole part and I would like to thank Enrico Calloni and Luigi Rosa for the Casimir effect part.

Data Availability Statement This manuscript has no associated data or the data will not be deposited. [Authors' comment: This is a theoretical study and no experimental data has been listed.]

Open Access This article is distributed under the terms of the Creative Commons Attribution 4.0 International License (http://creativecomm ons.org/licenses/by/4.0/), which permits unrestricted use, distribution, and reproduction in any medium, provided you give appropriate credit to the original author(s) and the source, provide a link to the Creative Commons license, and indicate if changes were made.

Funded by SCOAP ${ }^{3}$.

\section{A A particular case: transforming a singularity into a TW}

As a sub-case of the solution (25), we consider the following assumption on the throat radius:

$r_{0}=r_{1}$

representing the EB wormhole shape function. Plugging $b(r)=r_{0}^{2} / r$ and Eq. (3) into Eq. (8), we find the following form for the redshift function:

$\phi(r)=-\frac{1}{2} \ln \left(1-\frac{r_{0}^{2}}{r^{2}}\right)+\phi\left(r_{0}\right)$,

with the usual replacement of $a$ with $r$. We find that for $r=r_{0}$ we have neither a black hole nor a traversable wormhole, but we have a singularity described by the following line element: $d s^{2}=-\frac{d t^{2}}{1-\frac{r_{0}^{2}}{r^{2}}}+\frac{d r^{2}}{1-\frac{r_{0}^{2}}{r^{2}}}+r^{2} d \Omega^{2}$.

The metric (104) has been obtained also in Ref. [14] with $\alpha=-1$ and $\omega=3$, out of the phantom region. Because of (33), $p_{t}(r)$ is divergent when $r \rightarrow r_{0}$; indeed from the line element (104), we find [15]

$p_{t}(r)=\frac{\left(3 r^{2}-r_{0}^{2}\right) r_{0}^{2}}{r^{4}\left(r^{2}-r_{0}^{2}\right)}$

The presence of a singularity for the metric (104) is also confirmed by the calculation of the Kretschmann scalar,

$R_{\alpha \beta \gamma \delta} R^{\alpha \beta \gamma \delta}=8 \frac{r_{0}^{4}\left(7 r^{4}-8 r^{2} r_{0}^{2}+3 r_{0}^{4}\right)}{\left(r-r_{0}\right)^{2}\left(r+r_{0}\right)^{2} r^{8}}$.

Nevertheless, instead of discarding the metric (104), we can use the strategy adopted in Refs. [11,34] and we slightly modify the line element (104) in the following way:

$d s^{2}=-\frac{d t^{2}}{1-\frac{\lambda r_{0}^{2}}{r^{2}}}+\frac{d r^{2}}{1-\frac{r_{0}^{2}}{r^{2}}}+r^{2} d \Omega^{2}, \quad \lambda \in[0,1]$.

Of course, we could have adopted the following distortion of the metric (104):

$d s^{2}=-\frac{d t^{2}}{1-\frac{r_{0}^{2}}{r^{2}}+\lambda}+\frac{d r^{2}}{1-\frac{r_{0}^{2}}{r^{2}}}+r^{2} d \Omega^{2}$,

like in Refs. [11,34]. However, the line element (107) has the advantage of interpolating between the singularity, when $\lambda=$ 1 , and the EB wormhole when $\lambda=0$, while the line element (108) does not have this property. This simple modification produces the following effects on the SET:

$T_{\mu \nu}=\operatorname{diag}\left(\rho(r), p_{r}^{\lambda}(r), p_{t}^{\lambda}(r), p_{t}^{\lambda}(r)\right)$,

where

$\rho(r)=-\frac{r_{0}^{2}}{\kappa r^{4}}$,

$p_{r}^{\lambda}(r)=-\frac{r_{0}^{2}}{\kappa r^{4}} \frac{r^{2}(2 \lambda+1)-3 \lambda r_{0}^{2}}{r^{2}-\lambda r_{0}^{2}}$,

$p_{t}^{\lambda}(r)=\frac{r_{0}^{2}}{\kappa r^{4}} \frac{(2 \lambda+1) r^{4}+r_{0}^{2} \lambda(\lambda-5) r^{2}+r_{0}^{4} \lambda^{2}}{\left(r^{2}-\lambda r_{0}^{2}\right)^{2}}$.

In proximity of the throat, we find

$\rho(r) \underset{r \rightarrow r_{0}}{\rightarrow}-\frac{1}{\kappa r_{0}^{2}}$, 
$p_{r}^{\lambda}(r) \underset{r \rightarrow r_{0}}{\rightarrow}-\frac{1}{\kappa r_{0}^{2}}$

$p_{t}^{\lambda}(r) \underset{r \rightarrow r_{0}}{\rightarrow} \frac{2 \lambda^{2}-3 \lambda+1}{\kappa r_{0}^{2}(1-\lambda)^{2}}$

and, for small $\lambda$, we find

$p_{r}^{\lambda}(r) \simeq-\frac{r_{0}^{2}}{\kappa r^{4}}-\frac{2 \lambda r_{0}^{2}}{\kappa r^{4}}+\frac{2 r_{0}^{4} \lambda}{\kappa r^{6}}+O\left(\lambda^{2}\right)$,

$p_{t}^{\lambda}(r) \simeq \frac{r_{0}^{2}}{\kappa r^{4}}+\frac{2 \lambda r_{0}^{2}}{\kappa r^{4}}-\frac{3 r_{0}^{4} \lambda}{\kappa r^{6}}+O\left(\lambda^{2}\right)$.

If we assume that the relationship $r_{0}=r_{1}$ holds, one finds

$\frac{r_{0}^{2}}{\kappa}=\frac{\hbar c \pi^{2}}{720}$

i.e. we have the Casimir coefficients. Note that

$\lim _{\lambda \rightarrow 0} \lim _{r \rightarrow r_{0}} T_{\mu \nu}=\lim _{r \rightarrow r_{0}} \lim _{\lambda \rightarrow 0} T_{\mu \nu}$,

while

$\lim _{\lambda \rightarrow 1} \lim _{r \rightarrow r_{0}} T_{\mu \nu} \neq \lim _{r \rightarrow r_{0}} \lim _{\lambda \rightarrow 1} T_{\mu \nu}$

Indeed

$$
\begin{aligned}
& \frac{1}{\kappa r_{0}^{2}} \lim _{\lambda \rightarrow 1} \operatorname{diag}\left(-1,-1, \frac{2 \lambda-1}{\lambda-1}, \frac{2 \lambda-1}{\lambda-1}\right) \\
& \quad \neq \frac{1}{\kappa r_{0}^{2}} \lim _{r \rightarrow r_{0}} \operatorname{diag}\left(-1,-3,1-\frac{2 r_{0}^{2} r^{2}}{\left(r^{2}-r_{0}^{2}\right)^{2}}, 1-\frac{2 r_{0}^{2} r^{2}}{\left(r^{2}-r_{0}^{2}\right)^{2}}\right)
\end{aligned}
$$

and the divergence persists in $p_{t}(r)$ but with a different behavior. The same problem appears in Ref. [34], where the authors examine a line element of the form

$d s^{2}=-\left(1-\frac{r_{0}}{r}+\lambda\right) d t^{2}+\frac{d r^{2}}{1-\frac{r_{0}}{r}}+r^{2}\left(d \theta^{2}+\sin ^{2} \theta d \varphi^{2}\right)$,

leading to the following SET:

$$
\begin{aligned}
T_{\mu \nu}= & \frac{r_{0} \lambda}{\kappa r^{2}\left(\lambda r+r-r_{0}\right)} \\
& \times \operatorname{diag}\left(0,-1, \frac{2 \lambda r+2 r-r_{0}}{4\left(\lambda r+r-r_{0}\right)}, \frac{2 \lambda r+2 r-r_{0}}{4\left(\lambda r+r-r_{0}\right)}\right) .
\end{aligned}
$$

It is easy to check that, even in this case, a non-commutative behavior appears. Indeed

$$
\begin{aligned}
\lim _{\lambda \rightarrow 0} T_{\mu \nu} & =\frac{1}{\kappa r_{0}} \operatorname{diag}\left(0,-\frac{1}{r_{0}}, \frac{\lambda+1}{2 \lambda r_{0}}, \frac{\lambda+1}{2 \lambda r_{0}}\right) \\
& \neq \lim _{r \rightarrow r_{0}} \lim _{\lambda \rightarrow 0} T_{\mu \nu}=\frac{1}{\kappa r_{0}} \operatorname{diag}(0,0,0,0) .
\end{aligned}
$$

\section{References}

1. H. Casimir, Proc. Kon. Ned. Akad. Wetenschap 51, 793 (1948)

2. M. Sparnaay, Nature 180, 334 (1957)

3. M. Sparnaay, Physica 24, 751 (1958)

4. A. van Silfhout, Dispersion Forces between Macroscopic Objects. Drukkerij Holland, N.V., Amsterdam, 1966

5. S. Lamoreaux, Phys. Rev. Lett. 78, 5 (1997)

6. G. Bressi, G. Carugno, R. Onofrio, G. Ruoso, Phys. Rev. Lett. 88, 041804 (2002). arXiv:quant-ph/0203002

7. T. Boyer, Phys. Rev. 174, 1764 (1968)

8. S.K. Blau, M. Visser, A. Wipf, Nucl. Phys. B 310, 163 (1988)

9. D. Hochberg, Phys. Lett. B 268, 377 (1991)

10. M.S. Morris, K.S. Thorne, Am. J. Phys. 56, 395 (1988)

11. M. Visser, Lorentzian Wormholes: From Einstein to Hawking (American Institute of Physics, New York, 1995)

12. M.S. Morris, K.S. Thorne, U. Yurtsever, Phys. Rev. Lett. 61, 1446 (1988)

13. L.H. Ford, T.A. Roman, Phys. Rev. D 53, 5496 (1996). arXiv:gr-qc/9510071

14. F.S.N. Lobo, Phys. Rev. D 71, 084011 (2005). arXiv:gr-qc/0502099

15. S.W. Kim, J. Korean Phys. Soc. 63, 1887 (2013). arXiv:1302.3337 [gr-qc]

16. P.W. Milonni, The Quantum Vacuum: An Introduction to Quantum Electrodynamics (Academic Press Inc., New York, 1994)

17. M. Bouhmadi-L opez, A. Errahmani, P. Mart n-Moruno, T. Ouali, Y. Tavakoli, Int. J. Mod. Phys. D 24(10), 1550078 (2015). arXiv:1407.2446 [gr-qc]

18. P. Martin-Moruno, M. Visser, JHEP 1309, 050 (2013). arXiv:1306.2076 [gr-qc]

19. M. Bouhmadi-Lopez, F.S.N. Lobo, P. Martin-Moruno, JCAP 1411, 007 (2014). arXiv:1407.7758 [gr-qc]

20. M. Barriola, A. Vilenkin, Phys. Rev. Lett. 63, 341 (1989)

21. M. Visser, S. Kar, N. Dadhich, Phys. Rev. Lett. 90, 201102 (2003). arXiv:gr-qc/0301003

22. S.V. Sushkov, Phys. Rev. D 71, 043520 (2005). arXiv:gr-qc/0502084

23. F.S.N. Lobo, Phys. Rev. D 71, 084011 (2005). arXiv:gr-qc/0502099

24. F.S.N. Lobo, Phys. Rev. D 71, 124022 (2005). arXiv:gr-qc/0506001

25. R. Garattini, Class. Quantum Gravity 22, 1105 (2005). arXiv:gr-qc/0501105

26. R. Garattini, Class. Quantum Gravity 24, 1189 (2007). arXiv:gr-qc/0701019

27. R. Garattini, F.S.N. Lobo, Class. Quantum Gravity 24, 2401 (2007). arXiv:gr-qc/0701020

28. R. Garattini, F.S.N. Lobo, Phys. Lett. B 671, 146 (2009). arXiv:0811.0919 [gr-qc]

29. R. Garattini, F.S.N. Lobo, Phys. Rev. D 85, 024043 (2012). arXiv:1111.5729 [gr-qc]

30. H. Ellis, J. Math. Phys. 14, 104 (1973)

31. K.A. Bronnikov, Acta Phys. Pol. B 4, 251 (1973)

32. P. Mishra, T.P. Singh, Phys. Rev. D 88, 104036 (2013). arXiv:1108.2375 [astro-ph.GA]

33. K.K. Nandi, Y.Z. Zhang, R.G. Cai, A. Panchenko, Phys. Rev. D 79, 024011 (2009). arXiv:0809.4143 [gr-qc]

34. T. Damour, S.N. Solodukhin, Phys. Rev. D 76, 024016 (2007). arXiv:0704.2667 [gr-qc] 